\title{
On Electro- and Thermomigration and Thermoelectric Transport Coefficients in Metals
}

\author{
H.-J. Bohn and G. Simon \\ Institut für Theoretische Physik der Technischen Universität Braunschweig
}

Z. Naturforsch. 37 a, 1327-1332 (1982); received August 21, 1982

\begin{abstract}
The system of coupled Boltzmann transport equations for electrons, phonons and impurities in metals is set up and solved with special interest for the effect of the cross-coupling terms. General expressions for the effective charge in electromigration and heat of transport in thermomigration are given. The cross-coupling terms in the transport equations lead to a number of corrections in the thermoelectric transport coefficients. Noteworthy is the result that the resistance by impurities depends now on the temperature. The investigation is restricted to high temperatures, isotropic dependence of the energies of electrons and phonons on wave number vector, and to normal scattering processes of phonons.
\end{abstract}

\section{Introduction}

Electro- and thermotransport of impurity atoms in metals is mainly caused by impact of electrons $[1,2]$ and of phonons [3]. To treat the problem in a general way, we consider the system as a gas mixture of impurity atoms, electrons and phonons [3]. The transport problem is formulated within the framework of conventional Boltzmann theory. If $f(\boldsymbol{k}, \boldsymbol{r}), g(\boldsymbol{q}, \boldsymbol{r})$ and $s(\boldsymbol{p}, \boldsymbol{r})$ are respectively the distribution functions for electrons, phonons and impurity atoms the transport equations are given by

$$
\begin{aligned}
& \left.\frac{\partial f(\boldsymbol{k}, \boldsymbol{r}, t)}{\partial t}\right|_{\text {FIELD }}=\left.\frac{\partial f}{\partial t}\right|_{\text {COLL }} \\
& =F^{\mathrm{ee}}(f, f)+F^{\mathrm{ep}}(f, g)+F^{\mathrm{ei}}(f, s), \\
& \left.\frac{\partial g(\boldsymbol{q}, \boldsymbol{r}, t)}{\partial t}\right|_{\text {FIELD }}=\left.\frac{\partial g}{\partial t}\right|_{\text {COLL }} \\
& =F^{\mathrm{pe}}(g, f)+F^{\mathrm{pp}}(g, g)+F^{\mathrm{pi}}(g, s), \\
& \left.\frac{\partial s(\boldsymbol{p}, \boldsymbol{r}, t)}{\partial t}\right|_{\text {FIELD }}=\left.\frac{\partial s}{\partial t}\right|_{\text {COLL }} \\
& =F^{\mathrm{ie}}(s, f)+F^{\mathrm{ip}}(s, g)+F^{\mathrm{ii}}(s, s) .
\end{aligned}
$$

The left hand sides of these equations are the field terms and the right hand sides the scattering terms. These are functionals of the variables indicated.

The transport equations (1.1) to (1.3) are usually solved with the assumption that phonons and im-

Reprint requests to Prof. Dr. G. Simon, Institut für Theoretische Physik der Technischen Universität Braunschweig, Mendelssohnstrasse 3, D-3300 Braunschweig. purity atoms can be considered to be in equilibrium. Without this assumption the solution of the transport equation leads not only to the desired coefficients of electro- and thermotransport but also - as a byproduct - to generalized expressions for the dc thermoelectric transport coefficients of metals. This is the aim of this paper. As one of the results of the generalized transport coefficients we find that the electrical resistivity which is due to the impurity atoms depends on the temperature. More precisely, the distribution function $g(\boldsymbol{q}, \boldsymbol{r})$, appearing in (1.1) for the conduction electrons depends via (1.2) and (1.3) on the distribution function $s(\boldsymbol{p}, \boldsymbol{r})$ of the impurities. This induces indirectly temperature dependent terms for the resistivity by impurities.

General expressions for electro- and thermomigration $[4,5,6,7]$ and de thermoelectric coefficients $[8,9]$ are obtained.

In this paper we consider only the situation at high temperatures. The case of low temperatures will be treated in a separate publication [10].

The present paper is arranged as follows. In Chapter 2 the interactions and the collision integrals are described. The solution of the transport equations is essentially straightforward but tedious. The main points of it are indicated in Chapter 3 without going into too much details. Finally the results of the calculations are given and discussed in Chapter 4 . Moreover in order not to burden the formulae too much the utmost tolerable simplifications are made (only one conduction band, spherical Fermi surface, only one phonon mode and so on). 


\section{Interactions and Collision Integrals}

Only one-phonon transitions are taken into account for the interactions of the phonons with electrons [11,12] and with impurities [13], furthermore only normal processes. This may turn out to be a serious restriction. The electron-impurity interaction is described by hard-sphere-scattering. The collision terms in (1.1) to (1.3) are then

$$
\begin{aligned}
& F^{\mathrm{ep}}(f, g)=\frac{\Omega}{(2 \pi)^{3} \hbar} \iint_{\boldsymbol{q}}\left|\boldsymbol{M}_{\boldsymbol{k}, \boldsymbol{q}}^{\mathrm{pe}}\right|^{2}\left(\delta\left(\varepsilon_{\boldsymbol{k}-\boldsymbol{q}}^{\mathrm{e}}-\varepsilon_{\boldsymbol{k}}^{\mathrm{e}}+\hbar \omega_{q}\right)\left\{f_{\boldsymbol{k}-\boldsymbol{q}}\left(1-f_{k}\right) g_{q}-f_{k}\left(1-f_{k-q}\right)\left(1+g_{q}\right)\right\}\right. \\
& \left.+\delta\left(\varepsilon_{\boldsymbol{k}+q}^{\mathrm{e}}-\varepsilon_{\boldsymbol{k}}^{\mathrm{e}}-\hbar \omega_{q}\right)\left\{f_{k+q}\left(1-f_{k}\right)\left(1+g_{q}\right)-f_{k}\left(1-f_{k+q}\right) g_{q}\right\}\right) \mathrm{d}^{3} q, \\
& F^{\mathrm{ei}}(f, s)=\frac{\hbar}{4 m} \iiint_{\boldsymbol{P}} \int_{\boldsymbol{e}} \sigma_{\mathrm{ei}}\left|\boldsymbol{e} \cdot\left(\boldsymbol{k}-\frac{m}{\boldsymbol{M}} \boldsymbol{p}\right)\right|\left\{s^{\prime} f^{\prime}(1-f)-s f\left(1-f^{\prime}\right)\right\} \mathrm{d}^{2} e \mathrm{~d}^{3} p, \\
& F^{\mathrm{pe}}(g, f)=\frac{\Omega}{2(2 \pi)^{3} \hbar} \iiint_{\boldsymbol{k}}\left|M_{k, q}^{\mathrm{pe}}\right|^{2}\left(\delta\left(\varepsilon_{\boldsymbol{k}-\boldsymbol{q}}^{\mathrm{e}}-\varepsilon_{\boldsymbol{k}}^{\mathrm{e}}+\hbar \omega_{q}\right)\left\{f_{k}\left(1-f_{k-q}\right)\left(1+g_{q}\right)-f_{k-q}\left(1-f_{k}\right) g_{q}\right\}\right. \\
& \left.+\delta\left(\varepsilon_{\boldsymbol{k}+\boldsymbol{q}}^{\mathrm{e}}-\varepsilon_{\boldsymbol{k}}^{\mathrm{e}}-\hbar \omega_{q}\right)\left\{f_{\boldsymbol{k}+\boldsymbol{q}}\left(1-f_{k}\right)\left(1+g_{q}\right)-f_{k}\left(1-f_{k+q}\right) g_{q}\right\}\right) \mathrm{d}^{3} k, \\
& F^{\mathrm{pi}}(g, s)=\frac{\Omega}{2(2 \pi)^{3} \hbar} \iint_{\boldsymbol{P}}\left|\boldsymbol{M}_{\boldsymbol{P}, \boldsymbol{q}}^{\mathrm{pi}}\right|^{2}\left(\delta\left(\varepsilon_{\boldsymbol{P}-\boldsymbol{q}}^{\mathrm{i}}-\varepsilon_{\boldsymbol{p}}^{\mathrm{i}}+\hbar \omega_{\boldsymbol{q}}\right)\left\{s_{\boldsymbol{p}-\boldsymbol{q}} g_{\boldsymbol{q}}-s_{\boldsymbol{p}}\left(1+g_{\boldsymbol{q}}\right)\right\}+\delta\left(\varepsilon_{\boldsymbol{p}+\boldsymbol{q}}^{\mathrm{i}}-\varepsilon_{\boldsymbol{p}}^{\mathrm{i}}+\hbar \omega_{\boldsymbol{q}}\right)\right. \\
& \left.\cdot\left\{s_{\boldsymbol{p}+\boldsymbol{q}}\left(1+g_{\boldsymbol{q}}\right)-s_{\boldsymbol{p}} g_{\boldsymbol{q}}\right\}\right) \mathrm{d}^{3} p, \\
& F^{\mathrm{ie}}(s, f)=\frac{\hbar}{2(2 \pi)^{3} m} \iiint_{\boldsymbol{k}} \iint_{\boldsymbol{e}} \sigma_{\mathrm{ei}}\left|\boldsymbol{e}\left(\boldsymbol{k}-\frac{m}{M} \boldsymbol{p}\right)\right|\left\{s^{\prime} f^{\prime}(1-f)-s f\left(1-f^{\prime}\right)\right\} \mathrm{d}^{2} e \mathrm{~d}^{3} k, \\
& F^{\mathrm{ip}}(s, g)=\frac{\Omega}{(2 \pi)^{3} \hbar} \iint_{\boldsymbol{k}} \int\left|\boldsymbol{M}_{\boldsymbol{P}, \boldsymbol{q}}^{\mathrm{pi}}\right|^{2}\left(\delta\left(\varepsilon_{\boldsymbol{P}-\boldsymbol{q}}^{\mathrm{i}}-\varepsilon_{\boldsymbol{P}}^{\mathrm{i}}+\hbar \omega_{\boldsymbol{q}}\right)\left\{s_{\boldsymbol{P}-\boldsymbol{q}} g_{\boldsymbol{q}}-s_{\boldsymbol{q}}\left(1+g_{\boldsymbol{q}}\right)\right\}+\delta\left(\varepsilon_{\boldsymbol{P}+\boldsymbol{q}}^{\mathrm{i}}-\varepsilon_{\boldsymbol{P}}^{\mathrm{i}}-\hbar \omega_{\boldsymbol{q}}\right)\right. \\
& \left.\cdot\left\{s_{\boldsymbol{p}+\boldsymbol{q}}\left(1+g_{\boldsymbol{q}}\right)-s_{\boldsymbol{p}} g_{\boldsymbol{q}}\right\}\right) \mathrm{d}^{3} q .
\end{aligned}
$$

$\Omega$ Volume of a unit cell, $m$ effective mass of the electrons, $M$ mass of an impurity atom, $\sigma_{\mathrm{ei}}$ scattering cross section for electron-impurity scattering, $\omega_{\boldsymbol{q}}$ frequency of a phonon with wave number $\boldsymbol{q}$, $\varepsilon_{\boldsymbol{k}}^{\mathrm{e}}$ energy of an electron with wave number $\boldsymbol{k}$, $\varepsilon_{p}^{i}$ energy of an impurity with wave number $\boldsymbol{p}$, $\hbar$ Planck's constant, $k_{\mathrm{B}}$ Boltzmann's constant, $T$ temperature, $n_{\mathrm{i}}$ number density of impurity atoms, $n_{\mathrm{h}}$ number density of host lattice atoms.

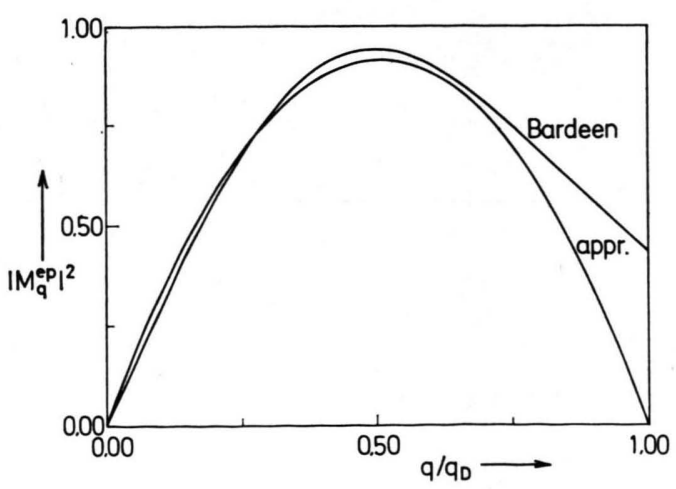

Fig. 1. Bardeen's matrix element and it's approximation (5) in dependence on the wave number $q / q_{\mathrm{D}}$.
The primed distribution functions are the distribution functions after the collision.

For the electron-phonon interaction Bardeen's matrix-element is used, but the quite complicated $q$-dependence of this matrix-element is replaced simply by (see Figure 1).

$$
\begin{aligned}
\left|M_{\boldsymbol{k}, \boldsymbol{q}}^{\mathrm{pe}}\right|^{2} & =\left|M_{\boldsymbol{q}}^{\mathrm{pe}}\right|^{2}=D \frac{q}{q_{\mathrm{D}}}\left(1-\frac{q}{q_{\mathrm{D}}}\right), \\
D & =\frac{2 \hbar \varepsilon_{\mathrm{F}}^{2} q_{\mathrm{D}}}{9 M^{\prime} c_{1} n_{\mathrm{e}} \Omega}
\end{aligned}
$$

$q_{\mathrm{D}}$ Debye wave number, $\varepsilon_{\mathrm{F}}$ Fermi energy, $M^{\prime}$ mass of a host atom, $c_{1}$ velocity of sound, $n_{\mathrm{e}}$ number density of the electrons.

For the phonon-impurity coupling we choose the matrix-element according to Maradudin [13]:

$$
\begin{aligned}
\left|M_{\boldsymbol{p}, \boldsymbol{q}}^{\mathrm{pi}}\right|^{2} & =\left|M_{\boldsymbol{q}}^{\mathrm{pi}}\right|^{2}=P \frac{q^{4}}{q_{\mathrm{D}}^{4}} N^{-1}(\beta, q) \\
P & =4 \pi \frac{\Omega \hbar^{4} q_{\mathrm{D}}^{7}}{M^{2}} \beta^{2}, \\
N(\beta, q) & =\left(1+3 \beta \frac{q^{2}}{q_{\mathrm{D}}^{2}}\right)^{2}+\frac{9 \pi^{2} q^{6}}{4 q_{\mathrm{D}}^{6}} \beta^{2}, \\
\beta & =\frac{M-M^{\prime}}{M^{\prime}} .
\end{aligned}
$$


(In (6) only the mass difference is taken into account, for contributions of changes in force and lattice constants see [15].)

The self-interactions of the impurity atoms can of course be neglected and at high temperatures only the self-interaction of the phonons is needed. This is represented by a relaxation process with relaxation time $\tau_{\mathrm{p}}$.

\section{Solution of the Transport equations}

The transport equations are treated in the usual manner. The collision integrals are identically zero for the equilibrium distributions of the particles. The driving forces - electrical field $E_{x}$ and temperature gradient $\mathrm{d} T / \mathrm{d} x$ - are supposed to be in $x$-direction. The distribution functions are expanded up to first order, that is:

$$
\begin{aligned}
& f(\boldsymbol{k})=f_{0}(k)-k_{x} \frac{\partial f_{0}}{\partial \varepsilon_{k}^{\mathrm{e}}} \Gamma\left(k ; E_{x}, \frac{\mathrm{d} T}{\mathrm{~d} x}\right), \\
& g(\boldsymbol{q})=g_{0}(q)+q_{x} \Psi\left(q ; E_{x}, \frac{\mathrm{d} T}{\mathrm{~d} x}\right), \\
& s(\boldsymbol{p})=s_{0}(p)\left(1+p_{x} \Lambda\left(p ; E_{x}, \frac{\mathrm{d} T}{\mathrm{~d} x}\right)\right)
\end{aligned}
$$

and the right hand side of equations (1.1) to (1.3) is linearized in the deviations of the distribution functions from equilibrium. The equilibrium distribution functions are known (Fermi, Bose and Maxwell-Boltzmann).

It is assumed that equilibrium is locally established. Then within linear response theory the field terms in the transport equations depend only on the local equilibrium distribution functions. The field terms can be expressed in a well known manner by the driving forces $E_{x}$ and $\mathrm{d} T / \mathrm{d} x$.

All these manipulations transform the transport equations into an inhomogeneous, linear system of integral equations. The inhomogeneous term is given by the driving forces

$$
\begin{aligned}
& \frac{D}{D t} f= k_{x} \frac{\hbar}{m} \frac{\partial f_{0}}{\partial \varepsilon_{\boldsymbol{k}}^{\mathrm{e}}} \\
& \cdot\left\{e E_{x}-\frac{1}{T} \frac{\mathrm{d} T}{\mathrm{~d} x}\left(T \frac{\mathrm{d} \varepsilon_{\mathrm{F}}}{\mathrm{d} T}+\varepsilon_{\boldsymbol{k}}^{\mathrm{e}}-\varepsilon_{\mathrm{F}}\right)\right\} \\
& \frac{D}{D t} g= q_{x} \frac{h c_{1}^{2}}{k_{\mathrm{B}} T} \\
& \cdot \frac{\exp \left(\hbar \omega_{\mathbf{q}} / k_{\mathrm{B}} T\right)}{\left(\exp \left(\hbar \omega_{\boldsymbol{q}} / k_{\mathrm{B}} T\right)-1\right)^{2}} \frac{1}{T} \frac{\mathrm{d} T}{\mathrm{~d} x},
\end{aligned}
$$

$$
\begin{aligned}
\frac{D}{D t} s= & p_{x} s_{0} \frac{\hbar}{M k_{\mathrm{B}} T} \\
& \cdot\left\{Z e E_{x}+\frac{1}{T} \frac{\mathrm{d} T}{\mathrm{~d} x}\right. \\
& \left.\cdot\left(\left(\varepsilon_{\boldsymbol{P}}^{\mathrm{i}}-V_{0}\right) \theta\left(\varepsilon_{\boldsymbol{P}}^{\mathrm{i}}-V_{0}\right)-\frac{5}{2} k_{\mathrm{B}} T\right)\right\} .
\end{aligned}
$$

In (8.3) $V_{0}$ is the maximum height of the lattice potential of the impurity atoms and $\theta(x)$ the unit step function. This takes care of the fact, that only impurity atoms with $\varepsilon_{p}^{i}>V_{0}$ can contribute to impurity migration.

In (1.1) and (1.3) the collision terms $F^{\text {ee }}$ and $F^{\text {ii }}$ are - as already mentioned - neglected. The remaining terms can be written as integrals:

$$
\begin{aligned}
& F^{\mathrm{ep}}=S_{12}^{\mathrm{p}}[\Psi]+S_{12}^{\mathrm{e}}[\Gamma], \\
& F^{\mathrm{ei}}=S_{13}^{\mathrm{i}}[\Lambda]+S_{13}^{\mathrm{e}}[\Gamma], \\
& F^{\mathrm{pe}}=S_{21}^{\mathrm{e}}[\Gamma]+S_{21}^{\mathrm{p}}[\Psi], \\
& F^{\mathrm{pp}}=S_{22}^{\mathrm{p}}[\Psi], \\
& F^{\mathrm{pi}}=S_{23}^{\mathrm{i}}[\Lambda]+S_{23}^{\mathrm{p}}[\Psi], \\
& F^{\mathrm{ie}}=S_{31}^{\mathrm{e}}[\Gamma]+S_{31}^{\mathrm{i}}[\Lambda], \\
& F^{\mathrm{ip}}=S_{32}^{\mathrm{p}}[\Psi]+S_{32}^{\mathrm{i}}[\Lambda] .
\end{aligned}
$$

These integrals can be calculated straightforward but are of some length, so we do not reproduce them here. The indices on the right-hand side are selfexplanatory.

If we go back to Bloch's assumption (phonon gas in equilibrium) and no impurity migration, then all integrals can be ignored except $S_{12}^{\mathrm{e}}[\Gamma]$ and $S_{13}^{\mathrm{e}}[\Gamma]$. For electron- and thermomigration the expressions $S_{31}^{\mathrm{e}}[\Gamma]$ and $S_{32}^{\mathrm{p}}[\Psi]$ describe "electron wind" and "phonon wind" respectively. Since impurity migration is naturally very slow, all terms depending on $\Lambda$ are negligible. The most important new feature is, that the deviation $\Psi$ of phonons from equilibrium is determined not solely by electron-phonon interaction, $S_{21}^{\mathrm{p}}[\Psi]$, but by phonon-impurity interaction, $S_{23}^{\mathrm{p}}[\Psi]$ as well.

Turning to the collision integrals in detail, the rather well known procedure of solution can be pursued. That is the energy-transfer in the collision processes can be taken to be small, which leads to relaxation time solutions. The system of transport equations reduces to a system of algebraic equations for the deviations $\Gamma, \Psi$ and $\Lambda$ coupled by integrals over these deviations. The solution of this system 
leads to integral equations with degenerate kernels, which can be solved analytically. The calculations are lengthy and we give only the results in the next section.

\section{Results and Discussion}

The quantities $J_{n}$ and $K_{n / 2}$ are given in the appendix.

(A) Electrical resistivity $\varrho$

constitutive equation: $j_{\mathrm{e}}=(1 / \varrho) E_{x}$

$$
\begin{aligned}
\varrho= & \left\{\varrho_{\mathrm{ph}}\left(1-20 J_{3}\right)+\varrho_{\mathrm{i}}\left(1-\delta \varrho_{\mathrm{i}}{ }^{\prime}\right)\right\} \\
& \cdot\left\{1-\delta \varrho_{\mathrm{i}}\right\}^{-1}, \\
\varrho_{\mathrm{ph}}= & \frac{3 \pi \Omega D k_{\mathrm{B}} T m^{1 / 2}}{202^{3 / 2} \hbar c_{1} \varepsilon_{\mathrm{F}}^{3 / 2} e^{2}} \frac{n_{x}}{n_{\mathrm{e}}}, \\
\varrho_{\mathrm{i}}= & \frac{\pi \sigma_{\mathrm{ei}} n_{\mathrm{i}}}{2^{3 / 2} e^{2} n_{\mathrm{e}}} \sqrt{m \varepsilon_{\mathrm{F}}}, \\
\delta \varrho_{\mathrm{i}}^{\prime}= & \frac{J_{1}}{3 \pi^{7 / 2} I_{1}}\left(K_{2}+12 \pi^{3} K_{1 / 2}+K_{0} \frac{Z_{\mathrm{eff}}^{\mathrm{ph}}}{Z_{\mathrm{eff}}^{\mathrm{el}}}\right. \\
\delta \varrho_{\mathrm{i}}= & \frac{4 J_{1} n_{\mathrm{i}}}{V^{\prime} \bar{\pi} I_{1} n_{\mathrm{e}}} K_{2} \frac{Z_{\mathrm{eff}}^{\mathrm{el}}}{Z_{\mathrm{eff}}^{\mathrm{ph}}}\left(1+F \pi_{3 / 2}^{3} K_{3 / 2} \frac{Z_{\mathrm{eff}}^{\mathrm{el}}}{Z_{\mathrm{eff}}^{\mathrm{ph}}}\right), \\
F= & \frac{1}{12 \pi^{3}} \frac{Z_{\mathrm{eff}}^{\mathrm{ph}}}{Z_{\mathrm{eff}}^{\mathrm{el}}} .
\end{aligned}
$$

(B) Thermopower $S$

constitutive equation:

$$
\begin{aligned}
& j_{\mathrm{e}}=\frac{1}{\varrho}\left(S-\frac{\mathrm{d} \varepsilon_{\mathrm{F}}}{|e| \mathrm{d} T}\right) \frac{1}{T} \frac{\mathrm{d} T}{\mathrm{~d} x}, \\
& S=-S_{0}\left\{1-\frac{1}{3} \frac{2 \varrho_{\mathrm{i}}+\left.\frac{\mathrm{d} \varrho_{\mathrm{i}}}{\mathrm{d} \varepsilon}\right|_{\varepsilon=1}}{\varrho}\right. \\
& \left.\quad+\left(S_{1}\left(1+\delta S_{1}\right)+S_{2}\right) /\left(1-\delta \varrho_{\mathrm{i}}\right)\right\}, \\
& S_{0}=\frac{\pi^{2} k_{\mathrm{B}}^{2} T}{|e| \varepsilon_{\mathrm{F}}}, \\
& S_{1}=\frac{2}{\pi^{2}} J_{2} \frac{\varepsilon_{\mathrm{F}}}{k_{\mathrm{B}} T} \frac{n_{x}}{n_{\mathrm{e}}}, \\
& \delta S_{1}=\frac{1}{5 \pi^{5 / 2}} K_{1 / 2} \frac{\varrho_{\mathrm{i}}}{\varrho_{\mathrm{ph}}}\left(1+F K_{0} / K_{1 / 2}\right),
\end{aligned}
$$

$$
\begin{aligned}
S_{2}= & \frac{4 J_{1} n_{\mathrm{i}} \varepsilon_{\mathrm{F}}}{\pi^{5 / 2} I_{1} n_{\mathrm{e}} k_{\mathrm{B}} T^{\prime}} K_{2} \frac{Z_{\mathrm{eff}}^{\mathrm{el}}}{Z_{\mathrm{eff}}^{\mathrm{ph}}} \frac{5}{2} \\
& \cdot\left(1+F K_{3 / 2} / K_{2}\right), \\
\frac{n_{x}}{n_{\mathrm{e}}}= & \left\{\begin{array}{l}
\text { ratio of number density of atoms to } \\
\text { electrons if }<4,4 \text { (for semi metals). }
\end{array}\right.
\end{aligned}
$$

(C) Heat conductivity $\lambda$

constitutive equation:

$$
w_{x}=-\lambda\left(\mathrm{d} T / d_{x}\right) \text { and } j_{\mathrm{e}}=0,
$$

electronic part:

$$
\begin{aligned}
\lambda_{\mathrm{e} l}= & \frac{\pi^{2} k_{\mathrm{B}}^{2}}{3 e^{2}} \frac{T}{\varrho}\left(1-L_{1}-L_{2}-L_{3}\right), \\
L_{1}= & 20 J_{3} \frac{\varrho_{\mathrm{ph}}}{\varrho_{\mathrm{ph}}+\varrho_{\mathrm{i}}}, \\
L_{2}= & 3 \frac{|e| S T}{\varepsilon_{\mathrm{F}}}, \\
L_{3}= & \left(\frac{Z|e| S}{k_{\mathrm{B}}}-\frac{5}{2}\right) \frac{8 J_{1} n_{\mathrm{i}} Z_{\mathrm{eff}}^{\mathrm{el}} k_{\mathrm{B}} T}{\sqrt{\pi} I_{1} n_{\mathrm{e}} Z_{\mathrm{eff}}^{\mathrm{ph}} \varepsilon_{\mathrm{F}}} K_{2} \\
& +\frac{4 J_{5} K_{1 / 2} n_{x} \varrho_{\mathrm{i}} k_{\mathrm{B}} T}{5 \sqrt{\pi} n_{\mathrm{e}} \varrho_{\mathrm{ph}} \varepsilon_{\mathrm{F}}} ;
\end{aligned}
$$

lattice part:

$$
\begin{aligned}
& \lambda_{1}=\frac{\pi^{2} k_{\mathrm{B}}^{2}}{3 e^{2}} \frac{T}{\varrho}\left(L_{4}+L_{5}\right) \\
& L_{4}=\frac{J_{6} n_{x}^{2} \varrho}{15 \pi^{3} n_{\mathrm{e}}^{2} \varrho_{\mathrm{ph}}}, \\
& L_{5}=\frac{K_{0} J_{5}^{2} n_{\mathrm{i}} n_{x}^{2} \varrho_{\mathrm{i}}}{900 \pi^{13 / 2} J_{1} n_{\mathrm{e}}^{3} \varrho_{\mathrm{ph}}} Z_{\mathrm{eff}}^{\mathrm{ph}}
\end{aligned}
$$

(D) Lorenz-number $L$

constitutive equation: $L=\lambda \varrho / T$,

$$
L=\frac{\pi^{2} k_{\mathrm{B}}^{2}}{3 e^{2}}\left(1-L_{1}-L_{2}-L_{3}+L_{4}+L_{5}\right) .
$$

(E) Electromigration

constitutive equation:

$$
\begin{aligned}
j_{\mathrm{i}} & =n_{\mathrm{i}} D_{\mathrm{u}} Z_{\mathrm{eff}} e E_{x} / k_{\mathrm{B}} T \\
Z_{\mathrm{eff}} & =Z_{\mathrm{eff}}^{\mathrm{el}}+\bar{Z}_{\mathrm{eff}}^{\mathrm{ph}}\left(1-E_{1} K_{0}\right)^{-1}-Z(1+\delta Z), \\
Z_{\mathrm{eff}}^{\mathrm{el}} & =\frac{n_{\mathrm{e}} \varrho_{\mathrm{i}}}{n_{\mathrm{i}} \varrho} \\
\bar{Z}_{\mathrm{eff}}^{\mathrm{ph}} & =Z_{\mathrm{eff}}^{\mathrm{ph}}\left(k_{\mathrm{B}} T / Q\right)^{3 / 2}
\end{aligned}
$$




$$
\begin{aligned}
Z_{\mathrm{eff}}^{\mathrm{ph}}= & \frac{3 \pi \Omega M^{3 / 2} c_{1} P n_{\mathrm{h}} J_{1}}{2^{3 / 2}\left(k_{\mathrm{B}} T\right)^{3 / 2} \hbar e^{2} n_{\mathrm{e}} \varrho}, \\
\delta Z= & \frac{J_{1} n_{\mathrm{i}} K_{2}}{3 \pi^{7 / 2} I_{1} n_{\mathrm{e}}\left(1-E_{1} K_{0}\right)} \\
& \cdot\left(Z_{\mathrm{eff}}^{\mathrm{el}}+Z_{\mathrm{eff}}^{\mathrm{ph}}\left(1-E_{1} K_{0}\right)^{-1}\right), \\
E_{1}= & \frac{1}{60 \pi^{9 / 2}} \frac{J_{4}}{I_{1} J_{1}} \frac{\varrho_{\mathrm{i}} Z_{\mathrm{eff}}^{\mathrm{ph}}}{\varrho_{\mathrm{ph}} Z_{\mathrm{eff}}^{\mathrm{el}}} ;
\end{aligned}
$$

$Q$ activation energy for impurity migration, $D_{\mathrm{u}}$ total diffusion constant $\left(D_{\mathrm{u}}=D_{0} \exp \left(-Q / k_{\mathrm{B}} T\right), e\right.$ charge of an electron, $Z$ excess valence of an impurity atom.

\section{(F) Thermomigration}

constitutive equation:

$$
\begin{aligned}
j_{\mathrm{i}}= & -\left(n_{\mathrm{i}} D_{\mathrm{u}} Q_{\mathrm{heat}} / k_{\mathrm{B}} T\right)(1 / T)(\mathrm{d} T / \mathrm{d} x) \\
& \text { and for closed circuits } j_{\mathrm{e}}=0 \\
Q_{\mathrm{heat}}= & Q_{\mathrm{tp}}+Q_{\mathrm{ph}}+Q_{\mathrm{va}}+Q_{\mathrm{ent}} \\
Q_{\mathrm{tp}}= & Z|e| T\left(S-\frac{\mathrm{d} \varepsilon_{\mathrm{F}}}{|e| \mathrm{d} T}\right) \\
Q_{\mathrm{ph}}= & \frac{J_{5} I_{1} \varrho}{10 J_{1} \varrho_{\mathrm{ph}}} Z_{\mathrm{eff}}^{\mathrm{ph}} k_{\mathrm{B}} T \\
Q_{\mathrm{va}}= & Q-V_{0} \\
Q_{\mathrm{ent}}= & -\frac{5}{2} k_{\mathrm{B}} T(1+\delta Z)
\end{aligned}
$$

The parts $\varrho_{\mathrm{ph}}$ and $\varrho_{\mathrm{i}}$ of the electrical resistivity are the well-known contributions from phonons and impurities, the factor $1-20 J_{3}$ is due to phonondrag. New are the terms $\delta \varrho_{i}^{\prime}$ and $\delta \varrho_{i}$. Both are small, $\delta \varrho_{i}{ }^{\prime}$ being still smaller than $\delta \varrho_{i}$. The latter one is observable in metallic alloys [14].

For the thermopower $S$ the first terms in curly brackets in (13) are the Nordheim-Gorter terms, $S_{1}$ is due to phonon-drag (already knowns), modified in our case by $\delta S_{1}$ and $\delta \varrho_{i}$ due to impurities. $S_{2}$ is a new contribution due simultaneously to impurities, electrons and phonons. A detailed comparison with experimental results, however, is not advisible, since we used isotropic distributions for electrons and phonons, and neglected umklapp processes. It is well known [9], that these assumptions are insufficient to treat thermopower. The additional term $\widetilde{S}$, caused by the cross-coupling terms in the transport equations, is

$$
\begin{aligned}
\tilde{S} & =S_{0} S_{1} \delta S_{1}+S_{0} S_{1} \delta \varrho_{\mathrm{i}}+S_{0} S_{2} \\
& =n_{\mathrm{i}}\left(\frac{a}{T}+b T^{3 / 2}+c \frac{T^{3 / 2}}{\varrho}\right)
\end{aligned}
$$

with constants $a, b, c$.
For the heat conductivity the terms $L_{2}, L_{3}, L_{4}$ and $L_{5}$ seem to be rather small and there may be a chance for observation only for the phonon-drag contribution $L_{1}$, which is of the order of 0.1 . It should be noted, that for noble metals $\varrho_{i}$ might at high temperatures be of the order of $\varrho \mathrm{ph}$.

In electromigration the electron wind contribution $Z_{\text {eff }}^{\text {el }}$ and the phonon wind contribution $\bar{Z}_{\text {eff }}^{\text {ph }}$ can be of the same order and have been described earlier [3]. In contrast to [3] we have used Maradudin's [13] matrix element for phonon-impurity scattering instead of Callaway's [15], which leads to better agreement with empirical values.

New features are a factor $\left(1-E_{1} K_{0}\right)^{-1}$ at $\bar{Z}_{\text {eff }}^{\mathrm{ph}}$, which is very close to unity and an additional term $Z \delta Z$. It is seen from (17) that this can also be thought of as a factor to $Z_{\text {eff }}^{\text {el }}+\bar{Z}_{\text {eff }}^{\text {ph }}$, deviating only a little from unity. But it should be noted, that this factor introduces a different dependence of $\boldsymbol{Z}_{\text {eff }}$ on $Z$.

For a unique determination of $Z_{\mathrm{eff}}^{\mathrm{el}}$ and $\bar{Z}_{\mathrm{eff}}^{\mathrm{ph}}$ it is necessary to know certain other transport coefficients, for example $\varrho_{i}$. Both contributions are inversely proportional to $\varrho$. A difficulty consists in the fact that the microscopic quantity $P$ (phononimpurity-interaction) cannot easily be connected to measurable macroscopic properties. Consistency in the determination of $\mathrm{P}$ can be checked in the small contribution of $\left(1-\delta \varrho_{i}\right)^{-1}$ to the electrical resistivity, in $\tilde{S}$ to the thermopower, in $L_{5}$ to heat conductivity (which is very small) and above all in $Q_{\mathrm{ph}}$ of thermomigration.

In thermomigration the vacancy contribution $Q_{\mathrm{va}}$ is as given in [2] and $[16,17]$. The term $Q_{\text {tp }}$ has been predicted by Huntington [2], but contrary to our result with $Z_{\text {eff }}$ instead of $Z$. This should be the case for closed circuits. Gerl [18] uses the specific thermopower instead of $S$.

The phononwind contribution $Q_{\mathrm{ph}}$ has been mentioned by Huntington [2], but not explicitly given. The last term $Q_{\text {ent }}$ is due to the enthalpy of the impurities.

Finally we would like to stress that it is desirable to establish and check relations between all of the transport coefficient for a system, including electroand thermomigration.

This work has been supported by the Deutsche Forschungsgemeinschaft and we acknowledge discussions on the subject with Th. Hehenkamp and H. Wever. 


\section{Appendix}

The quantities $J_{n}$ and $K_{n / 2}$ are defined by:

$$
\begin{aligned}
K_{n / 2} & =K_{n / 2}(c)=\int_{0}^{\infty} \frac{x^{n / 2} \exp (-x)}{c x^{3 / 2}+1} \mathrm{~d} x, \\
C & =\frac{J_{1} Z_{\mathrm{eff}}^{\mathrm{el}}}{I_{1} Z_{\mathrm{eff}}^{\mathrm{ph}}} . \\
I_{1} & =I_{1}(\beta)=\int_{0}^{1} \frac{u^{6}}{N(\beta, u)} \mathrm{d} u, \\
J_{1} & =J_{1}\left(\beta, b_{1}, b_{2}\right) \\
& =\int_{0}^{1} \frac{u^{6}-u^{7}}{N\left[1-u+b_{1}+b_{2}(u / N)\right]} \mathrm{d} u, \\
J_{2} & =J_{2}\left(b_{1}, b_{2}\right) \\
& =\int_{0}^{1} \frac{u^{2}-u^{3}}{\left[1-u+b_{1}+b_{2}(u / N)\right]} \mathrm{d} u, \\
J_{3} & =J_{3}\left(b_{1}, b_{2}\right) \\
& =\int_{0}^{1} \frac{u^{3}-2 u^{4}+u^{5}}{\left[1-u+b_{1}+b_{2}(u / N)\right]} \mathrm{d} u,
\end{aligned}
$$

[1] H. B. Huntington and A. R. Grone, J. Phys. Chem. Sol. 20, 76 (1961).

[2] H. B. Huntington, J. Phys. Chem. Sol. 29, 1641 (1968).

[3] H.-J. Bohn and G. Simon, Z. Naturforsch. 36a, 10 (1981).

[4] H. Wever, Elektro- und Thermotransport in Metallen, J. A. Barth, Leipzig 1973.

[5] L. J. Sham, Phys. Rev. 12 B, 3142 (1975).

[6] J. M. Schoen, J. Appl. Phys. 51, 508 (1980).

[7] R. S. Sorbello, Phys. Rev. 6 B, 4757 (1969).

[8] A. H. Wilson, Theory of Metals, University Press, Cambridge 1953.

[9] R. D. Barnard, Thermoelectricity of Metals and Alloys, Taylor \& Francis, London 1972.

$$
\begin{aligned}
J_{4} & =J_{4}\left(\beta, b_{1}, b_{2}\right) \\
& =\int_{0}^{1} \frac{u^{9}}{N^{2}\left[1-u+b_{1}+b_{2}(u / N)\right]} \mathrm{d} u \\
J_{5} & =J_{5}\left(\beta, b_{1}, b_{2}\right) \\
& =\int_{0}^{1} \frac{u^{5}}{N\left[1-u+b_{1}+b_{2}(u / N)\right]} \mathrm{d} u \\
J_{6} & =J_{6}\left(b_{1}, b_{2}\right) \\
& =\int_{0}^{1} \frac{u}{\left[1-u+b_{1}+b_{2}(u / N)\right]} \mathrm{d} u \\
u & =q / q_{\mathrm{D}}, \\
b_{1} & =\frac{2 \pi \hbar^{4} k_{\mathrm{B}} T}{\Omega m^{2} c_{\mathrm{e}} D \tau_{\mathrm{pr}}}, \\
\tau_{\mathrm{p}}^{-1} & =\tau_{\mathrm{pr}}^{-1} k_{\mathrm{B}} T u, \\
b_{2} & =\frac{n_{\mathrm{i}} M{ }^{1 / 2} P \hbar^{3}}{(2 \pi)^{3 / 2}\left(k_{\mathrm{B}} T\right)^{3 / 2} m^{2} D} .
\end{aligned}
$$

[10] H. J. Bohn and G. Simon, Z. Naturforsch. $\mathbf{3 7}$ a, 1333 (1982).

[11] A. Haug, Theoretische Festkörperphysik, F. Deuticke, Wien 1970.

[12] W. Brauer, Einführung in die Elektronentheorie der Metalle, Vieweg, Braunschweig 1966.

[13] A. A. Maradudin, in: Phonons and Phonon Interaction, ed.: T. A. Bak and W. A. Benjamin, New York 1964.

[14] Th. Hehenkamp, H.-J. Bohn, and G. Simon, to be published.

[15] J. Callaway, Nouvo Cim. 29, 883 (1963).

[16] J. A. Brinkman, Phys. Rev. 93, 345 (1954).

[17] A. D. LeClaire, Phys. Rev. 93, 344 (1954).

[18] M. Gerl, J. Phys. Chem. Sol. 28, 725 (1967). 